\title{
Shooting Pain
}

National Cancer Institute

\section{Source}

National Cancer Institute. Shooting Pain. NCI Thesaurus. Code C101180.

An intense sensation of discomfort or distress that radiates from one location to another sharply. 